$\mathbf{m} / \mathbf{S}$

médecine/sciences $1995 ; 11: 659-60$

\section{Vincent Lotteau}

\section{ADRESSE ET TIRÉS A PART}

V. Lotteau : chargé de recherche ì l'Inserm. Inserm U. 396, laboratoire d immunogénétique moléculaire, Instiţut biomédical des Cordeliers, 15, rue de l'Ecole de Médecine, 75006 Paris, France.

\title{
PRÉSENTATION DES ANTIGÉNES : QUI PRÉSENTE QUOI ?
}

$\mathbf{L}$

es lymphocytes $T$ porteurs d'un récepteur d'antigène $\alpha \beta$ sont activés par la reconnaissance de fragments peptidiques associés aux molécules du complexe majeur d'histocompatibilité $(\mathrm{CMH})$. Deux classes de molécules du CMH sont spécialisées dans la présentation de peptides de structure et d'origine différentes. L'occasion m'est donnée ici d'apporter quelques précisions sur cette dichotomie fonctionnelle qui pourrait ne plus apparaître évidente à la lecture d'un nombre grandissant d'articles.

Les molécules de classe I (CMH-I) présentent aux lymphocytes T CD8 cytotoxiques des antigènes endogènes synthétisés par la cellule présentatrice et dégradés en peptides dans le cytosol. Grâce à des transporteurs membranaires les peptides cytosoliques passent dans la lumière du réticulum endoplasmique où ils s'associent au CMH-I avant d'être exportés et exposés à la surface cellulaire. Dans certains cas, des antigènes exogènes induisent une bonne réponse cytotoxique. Il s'agit, en général, de micro-organismes ou d'antigènes particulaires internalisés dans des phagosomes puis transférés dans le cytosol [1]. Par la même voie de présentation, le $\mathrm{CMH}-\mathrm{I}$ peut donc déclencher une réponse cytotoxique contre des antigènes endogènes ou exogènes. Beaucoup moins bien documentée, la fixation de surface, sur le CMH-I, de peptides produits dans la voie d'endocytose et relargués dans le milieu extracellulaire pourrait être une voie alternative de présentation [2].

Les peptides cytosoliques transférés dans le réticulum endoplasmique, en admettant qu'ils aient les caractéris- tiques requises de taille et de séquence, ne se fixent pas aux molécules de classe II du CMH (CMH-II) car elles sont appariées à une molécule chaperon, la chaîne invariante $[3,4]$. ('elle-ci dirige le $\mathrm{CMH}-\mathrm{II}$ dans la voie d'endocytose où elle est elle-même rapidement dégradée. Les antigènes exogènes, internalisés par endocytose ou phagocytose, sont dégradés dans des compartiments vacuolaires où ils s'associent au ('MH-Il pour être présentés aux lýmphocytes T (:D4 auxiliaires. De très nombreux travaux ont conduit à l'identification de la voie d'endocitose comme site d'interaction entre peptides et ('MH-II mais il n'y a pas de consensus sur la distribution précise du ('MH-II et de la chaîne invariante dans les différents sous-compartiments de la voie d'endocytose, ni sur la route empruntée par le ('MH-II pour entrer ou sortir de ces organites. Par la microscopie électronique, le (MH-II, la chaîne invariante et les immunoglobulines internalisées furent d'abord localisés dans les endosomes précoces ; ces vésicules furent donc identifiées comme le lieu d'apprêtement des antigènes et de fixation des peptides sur le (:MH-II [5]. Par les mêmes techniques, d'autres groupes ont trouvé le ('MH-II essentiellement dans des prélysosomes pratiquement dépourvus de chaîne invariante $[6,7]$. Il fut alors suggéré que le ('MH-II se dirige directement du réseau transgolgien vers un compartiment particulier où il s'accumule et se charge de peptides. Plusieurs laboratoires, en utilisant les méthodes de fractionnement cellulaire, ont ensuite décrit une accumulation du CMH-II dans des vésicules d'endocytose différentes des endosomes précoces et tardifs et des lysosomes [8-12]. Il existe donc, 
d'après ces équipes, un compartiment spécialisé dans le chargement des peptides sur le CMH-II. Mais, là encore, rien n'est acquis car les caractéristiques de ce compartiment varient selon les études et certains mettent en doute la nécessité et l'existence même d'un compartiment spécialisé. Ils considèrent, au contraire, que le chargement des peptides sur le CMH-II doit se faire progressivement dès la sortie de l'appareil de ('olgi et à de multiples endroits dans des vésicules d'endocytose dont la relation avec les organites classiquement définis n'est pas toujours bien établie [13]. S. Amigorena ( $p .661$ de ce numéro) explique comment et pourquoi le chargement des peptides sur le CMH-II a lieu en un site unique et spécialisé dans des cellules B tumorales. Pour éviter les malentendus, les partisans et les détracteurs de cette théorie devraient donner une définition plus précise de ce qu'ils recherchent. Quelles sont les caractéristiques requises pour que des vésicules, visualisées par microscopie ou purifiées par fractionnement, soient considérées comme lieu de chargement des peptides sur le $\mathrm{CMH}$ II ? Quels autres éléments, en plus des peptides et du CMH-II, ces vésicules doivent-elles contenir pour que le chargement soit effectif ? La chaîne invariante, ou plutôt un fragment de chaîne invariante, devrait se trouver là pour au moins deux raisons. C'est elle qui dirige le transport intracellulaire du CMH-II par ses signaux de routage intracytoplasmique. C'est elle aussi qui maintient le CMH-II dans une conformation évitant son agrégation. La chaîne invariante est échangée contre des peptides qui jouent également un rôle important de prévention de l'agrégation du CMH-II. Enfin, HLA-DM (H2-M chez la souris) devrait se trouver aussi dans ces vésicules, ou à proximité, car il semble être un élément décisif permettant d'échanger un fragment de la chaîne invariante contre des peptides [14, 15]. Une minisynthèse rappelant les différentes fonctions hypothétiques de DM est présentée dans ce numéro. La production des peptides est peutêtre le chapitre le plus difficile à maîtriser car on ne connaît pas précisémment le lieu et les mécanismes de leur genèse. Comme pour le CMH-I, les peptides ne sont pas forcément pro- duits au site même de leur association avec le CMH-II. En outre, l'origine des peptides présentés par le CMH-II est multiple. Ils peuvent provenir de la dégradation des antigènes internalisés mais aussi des protéines synthétisées par la cellule présentatrice et ayant accès à la voie d'endocytose par d'autres mécanismes. L'autophagie, par exemple, est une voie de passage de matériel cytosolique vers les endosomes et les lysosomes. L'importance de la voie de présentation d'antigènes endogènes a été sous-estimée et on en sait peu de choses car elle est plus difficile à appréhender et les modèles d'étude sont rares et souvent artificiels. Le degré d'interconnection de ces deux voies de présentation reste à déterminer. Sont-elles liées, indépendantes ou complémentaires? Si les deux voies sont indépendantes, comme cela semble être parfois le cas, il serait tout à fait intéressant d'évaluer l'importance relative accordée à chacune d'elles par les différents types cellulaires. L'utilisation préférentielle de l'une ou l'autre voie est peut-être un dispositif primordial dans la mise en place du répertoire de l'immunité. Un autre aspect de la présentation antigénique qui stimule l'enthousiasme d'un nombre croissant d'immunologistes concerne la voie d'entrée des antigènes. Le système immunitaire doit être capable de reconnaître des quantités très faibles d'antigènes très différents. Bien que les macrophages effectuent en permanence une surveillance du milieu extra-cellulaire en prélevant des échantillons par pinocytose, un système plus efficace d'internalisation a été suggéré par l'intermédiaire de récepteurs comme ceux de la famille des récepteurs scavenger. Le dernier en date est le récepteur MARCO dont la structure rappelle un peu celle du collagène et qui est capable de fixer des bactéries à la surface de certains macrophages [16]. Aux dires des spécialistes, il est possible que le système immunitaire ait développé des dispositifs du même ordre, autres que celui des immunoglobulines, pour faciliter la pénétration et la présentation des antigènes solubles peu abondants. Ce domaine est en pleine expansion et nous réserve sans doute quelques surprises dans un avenir proche

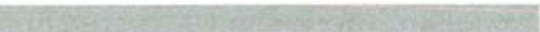

\section{RÉFÉRENCES}

1. Kovacsovics-Bankowski M, Rock KL. A phagosome to cytosol pathway for exogenous antigens presented on MHC class I molecules. Srience 1995 ; 267 : 243-6.

2. Harding CV, Song R. Phagocytic processing of exogenous particulate antigens by macrophages for presentation by class I MHC molecules. J Immunol 1994 ; 153 : 4925-33.

3. Rabourdin-Combe C, Bertolino P, CalinLaurens V, Gerlier D. La présentation de l'antigène aux lymphocytes $T$. médecine/ sciences $1991 ; 7: 674-80$.

4. Viville S, Rabourdin-Combe C. La chaîne invariante: son rôle et sa fonction dans la réponse immunitaire spécifique. médecine/ sciences $1994 ; 10: 163-70$.

5. Guargliardi LE, Koppelman B, Blum JS Marks MS, Creswell P, Brodsky FM. Colocalisation of molecules involved in antigen processing and presentation in an early endocytic compartment. Nature $1990 ; 343$ : 133-9. 6. Peters PJ, Neefjes IJ, Oorschot V, Ploegh HL Geuze HJ. Segregation of MHC class II molecules from MHCclass I molecules in the Golgi complex for transport to lysosomal compartments. Nature 1991 ; 349 : 669-76.

7. Neefjes II, Stollolrz V, Peters PJ, Geuze HJ, Ploegh HL. The biosynthetic pathway of MHC class II but not class I molecules intersects the endocytic route. (ell 1990;61 : 171-83.

8. Amigorena S, Drake J.R, Webster P, Mellman I. Transient accumulation of new class II MHC molecules in a novel endocytic compartment in B lymphocytes. Nature 1994 ; $369: 113-20$

9. Qiu Y, Xu X, Wandinger-Ness A, Dalke DP, Pierce SK. Separation of subcellular compartments containing distinct functional forms of MHC class II. I (ell Biol 1994 ; 125 : 595-605.

10. Tulp A, Verwoerd D. Dobberstein B, Ploegh HL, Pieters J. Isolation and characterization of the intracellular MHC class II compartment. Nature $1994 ; 369$ : 120-6.

11. W'est MA, Lucocg JM, W'atts C. Antigen processing and class II MHC peptide loading in human B Iymphoblastoid cells. Nature $1994 ; 369: 147-51$.

12. Rudensky AY, Maric M, Eastman S, Shoemaker L, De Roos PC, Blum J. Intracellular assembly and transport of endogenous peptide-MHC class If complexes. Immunity $1994 ; 1: 585-94$

13. Castellino F, Germain RN. Extensive trafficking of MHC class II-Invariant chain complexes in the endocytic pathway and appearance of peptide loaded class II in multiple compartments. Immunity $1995 ; 2$ : 73-88.

14. Morris P, Shaman J, Attaya M, Amaya M, Goodman S, Bergman C, Monacco.[J, Mellins E. An essential role for HLA-DM in antigen presentation by class II major histocompatibility molecules. Nature $1994 ; 368$ : 551-4.

15. Fling SP, Arp B, Pious D. HLA-DMA and $\mathrm{DMB}$ genes are both required for $\mathrm{MHC}$ class II/peptide complex formation in antigen presenting cells. Nature 1994 ; 368 : 55458. 16. Elomaa O, Kangas M, Sahlberg C, Tuukkanen J, Sormuner R, Liakka A, Thesieff l, Kraal G, Tryggnason K. Cloning of a novel bacteria binding receptor structurally related to scavenger receptor and expressed in a subset of macrophages. Cell 1995 ; 80 : 603-9. 